\title{
Status of uterine cervical dysplasia in District Ghaziabad, U.P; India
}

\author{
Sunita Vashist ${ }^{1}$, R. K. Arya ${ }^{2}$, Gajendra K. Gupta ${ }^{3, *}$, Narendra Singh $^{4}$, Raksha Arora $^{5}$ \\ ${ }^{\mathbf{1}}$ Assistant Professor, ${ }^{2}$ Professor and HOD, ${ }^{3}$ Professor, ${ }^{4}$ Associate Professor, Dept. of Community Medicine, Santosh Medical \\ College \& Hospital, Ghaziabad, Uttar Pradesh, ${ }^{5}$ Ex. Professor and HOD, Dept. of Obstetrics \& Gynaecology, Santosh Medical \\ College \& Hospital, Ghaziabad, Uttar Pradesh, India
}

*Corresponding Author:

Email: gajendrakgupta@gmail.com

\begin{abstract}
Introduction: Cancer of cervix is a common cancer that affects Indian women physically psychologically, socially and financially. The disease affects not just the women but also her family and society.

Aims and Objective: This study was designed to study status of uterine cervical dysplasia amongst women attending gynaec OPD of CHC Muradnagar, Ghaziabad, U.P.

Materials and Methods: This study was done at the community health centre (CHC), Muradnagar. It was a cross-sectional study done by using VIA (visual inspection using acetic acid) technique on uterine cervix on 1250 women aged above 30 years of age attending gynaecology OPD of CHC Muradnagar, Ghaziabad U.P. Purposive sampling was used to enroll all the women who were attending the gynaecology OPD at $\mathrm{CHC}$ and were coming in the eligibility criteria.

Result: Out of 1250 women 14 were found to be VIA positive, out of which 4 cases came out to be positive for dysplasia after doing biopsy under colposcopy. Three biopsy positive cases were found in the age group of 41 to 50 years and one case in age group of 51-60 years, two biopsy positive cases were found amongst participants who had no literacy and two were found positive amongst females who had at-least taken intermediate level or higher education, all the four biopsy positive cases were found amongst participants who were Hindu by religion, two biopsy positive cases were found amongst participants who were not working and two were found positive amongst females who were working, two biopsy positive cases were found amongst participants whose husbands were working as a professional and two cases were found positive amongst females whose husbands were working as either skilled/semi-skilled/unskilled labour or unemployed, two biopsy positive cases were found amongst participants who belonged to Class I Socio-economic status and two cases were found positive amongst females who belonged to Class III Socio-economic status.

Conclusion: Our epidemiological study revealed that many socio demographic variables such as increasing age, literacy status, religion, working status of participant and her husband, socio-economic status are important factors that significantly showed higher proportion of dysplasia that can influence development of cervical cancer. Also these factors are supported by several epidemiological studies as important risk factors for development of cervical cancer, specially done in low resource settings.

Recommendation: Mass health education and behaviour change interventions should be conducted at the community level in order to make all women of child bearing age aware about cervical cancer and its screening. The benefits of early diagnosis and treatment must be made aware to women so that they are encouraged to take cervical cancer screening.
\end{abstract}

Keywords: Cervical dysplasia, VIA screening, Socio-demographic variable, Socio-economic status.

\section{Introduction}

Cervical cancer is the commonest cancer causing death among women in developing countries. ${ }^{1}$ It is usually a very slow growing cancer that may not have symptoms for a long time but can be only detected in early stage with regular screening methods. $86 \%$ of all deaths due to cervical cancer are in developing countries, low and middle income countries. ${ }^{2,3}$ Cancer is leading cause of death in economically developed countries and the second leading cause of death in developing countries. ${ }^{4}$

Compared with other cancers, screening for cervical cancer is the most effective., ${ }^{5,6}$ Visual inspection of cervix after acetic acid application (VIA) has long been regarded as the most promising method for screening in resource -limited settings. VIA is performed by a trained health care provider who applies a $3 \%$ to $5 \%$ acetic acid solution to the cervix and then observes the transformation zone for 1-2 minutes for aceto-white epithelium, which is brought to be indicative of abnormal cellular changes. ${ }^{7,8}$
Many socio demographic variables such as increasing age, literacy status, religion, working status of participant and her husband, socio-economic status are important factors that significantly showed higher proportion of dysplasia that can influence development of cervical cancer. These factors are supported by several epidemiological studies as important risk factors for development of cervical cancer, specially done in low resource settings.

With the literature knowledge for cervical dysplasia we could gather the knowledge regarding this crucial disease of such a paramount importance. Also our objective was to generate awareness regarding importance of cervical cancer and its early detection through screening amongst all of the women attending CHC (community health centre) Muradnagar, Ghaziabad, UP, India and channelizing our study population towards early diagnosis, treatment and follow-up of cervical cancer. 


\section{Materials and Methods}

An observational cross sectional study was design, conducted at Community Health Center Muradnagar, situated at Community Development Block Muradnagar, Ghaziabad. Sample size as per the statistical method of 1250 was taken. As per the eligibility criteria all the women above 30 years of age attended gynaecology out patient department with gynaecological complaints were included in the study. All women aged below 30 years, with history of hysterectomy done, who had undergone cervical cancer treatment in past, currently pregnant women and who were not willing to give consent for the study were excluded from the study.

Information of individual and family was collected by pre- designed, pre-tested and structured schedule. Reproductive health related complaints were noted followed by clinical examination and cervical cancer screening. VIA (Visual inspection under acetic acid) of cervix was done, which came out to be VIA positive were send for biopsy to see dysplastic changes. The data collected was entered in MS Excel. After the master chart was prepared it was coded as categorical variables. The data was analyzed using SPSS version 17 (Statistical Package of Social Sciences) appropriate statistical test for the analysis of categorical variables was applied. Results were produced in form of frequencies and percentages.

\section{Result}

The majority of the participants of the study population were from rural area (67.7\%). Age group between 30 to 40 years was the most common age group in rural $(63.7 \%)$ as well as in urban population (59.9\%) followed by 41 to 50 age group. Very few participants were above 60 . Majority of the population were from Hindu background in rural (57.2\%) and from
Muslim background in urban $(51.2 \%)$ population. Almost half of the rural population $(50.2 \%)$ were illiterate whereas majority of the urban population $(59.7 \%)$ had some level of literacy. Majority of the participants from both rural (94.2\%) and urban $(92.8 \%)$ background were housewives and majority of their husbands were either skilled or semi-skilled workers. Majority of the participants' family per capita monthly income was less than Rs 5000 in both rural (87.8\%) and urban $(87.2 \%)$. Most of the families in both rural $(57.9 \%)$ and almost half of the urban $(50.5 \%)$ belonged to Class III of socio-economic status as per the Modified B.G Prasad scale (Revised for 2017) followed by Class II socio economic-status.

Out of 1250 cases 14 women were found to be VIA positive, out of which 4 cases came out to be positive for cervical dysplastic changes. Three biopsy positive cases were found in the age group of 41 to 50 years and one case in age group of 51-60 years, two biopsy positive cases were found amongst participants who had no literacy and two were found positive amongst females who had at-least taken intermediate level or higher education, all the four biopsy positive cases were found amongst participants who were Hindu by religion, two biopsy positive cases were found amongst participants who were not working and two were found positive amongst females who were working, two biopsy positive cases were found amongst participants whose husbands were working as a professional and two cases were found positive amongst females whose husbands were working as either skilled/semiskilled/unskilled labours or unemployed, two biopsy positive cases were found amongst participants who belonged to Class I Socio-economic status and two cases were found positive amongst females who belonged to Class III Socio-economic status.

Table-1: Distribution of the study population according to their Socio-Demographic characteristics

\begin{tabular}{|l|c|c|c|c|}
\hline \multicolumn{2}{|c|}{ Socio-Demographic Characteristics } & $\begin{array}{c}\text { Total } \\
(\mathbf{n = 1 2 5 0})\end{array}$ & $\begin{array}{c}\text { Biopsy } \\
\text { Positive }\end{array}$ & $\begin{array}{c}\text { Biopsy } \\
\text { Negative }\end{array}$ \\
\hline \multirow{4}{*}{ Age (Years) } & $30-40$ & $781(62.4 \%)$ & 0 & $781(100 \%)$ \\
\cline { 2 - 5 } & $41-50$ & $452(36.1 \%)$ & $3(0.7 \%)$ & $449(93.7 \%)$ \\
\cline { 2 - 5 } & $51-60$ & $15(1.2 \%)$ & $1(6.7 \%)$ & $14(93.3 \%)$ \\
\cline { 2 - 5 } & $61-70$ & $2(0.3 \%)$ & 0 & $2(100 \%)$ \\
\hline \multirow{4}{*}{ Religion } & Hindu & $680(54.4 \%)$ & $4(0.6)$ & $676(99.4)$ \\
\cline { 2 - 5 } & Muslim & $569(45.5 \%)$ & 0 & $569(100 \%)$ \\
\cline { 2 - 5 } & Others & $1(0.1 \%)$ & 0 & $1(100 \%)$ \\
\cline { 2 - 5 } & Illiterate & $588(7.0 \%)$ & $2(0.3 \%)$ & $586(99.7 \%)$ \\
\cline { 2 - 5 } & Primary School & $340(27.2 \%)$ & 0 & $340(100 \%)$ \\
\cline { 2 - 5 } & Middle School & $130(10.4 \%)$ & 0 & $130(100 \%)$ \\
\cline { 2 - 5 } & High School & $96(7.7 \%)$ & 0 & $96(100 \%)$ \\
\cline { 2 - 5 } & Intermediate & $52(4.2 \%)$ & $1(2 \%)$ & $51(98 \%)$ \\
\cline { 2 - 5 } & Graduate & $28(2.2 \%)$ & 0 & $28(100 \%)$ \\
\cline { 2 - 5 } & Post - Graduate & $16(1.3 \%)$ & $1(7 \%)$ & $15(93 \%)$ \\
\hline \multirow{4}{*}{$\begin{array}{l}\text { Occupation of the } \\
\text { Participant }\end{array}$} & Working & $78(6.2 \%)$ & $2(2.6 \%)$ & $76(97.4 \%)$ \\
\cline { 2 - 5 } & Non-Working & $1172(93.8 \%)$ & $2(0.2 \%)$ & $1170(99.8 \%)$ \\
\hline
\end{tabular}




\begin{tabular}{|l|c|c|c|c|}
\hline Husband's Occupation & Professional & $155(12.4 \%)$ & $2(1.3 \%)$ & $153(98,7 \%)$ \\
\cline { 2 - 5 } & $\begin{array}{c}\text { Others(Skilled } \\
\text { Worker/Semiskilled/Non } \\
\text {-skilled/Unemployed) }\end{array}$ & $1095(87.6 \%)$ & $2(0.2 \%)$ & $1093(99.8 \%)$ \\
\hline $\begin{array}{l}\text { Socio - Economic Status } \\
\text { (Modified B.G Prasad } \\
\text { Scale, 2017 Revision) }\end{array}$ & I & $24(1.9 \%)$ & $2(8.3 \%)$ & $22(91.7 \%)$ \\
\cline { 2 - 5 } & II & $433(34.6 \%)$ & 0 & $433(100 \%)$ \\
\cline { 2 - 5 } & III & $694(55.6 \%)$ & $2(0.3 \%)$ & $692(99.7 \%)$ \\
\cline { 2 - 5 } & IV & $99(7.9 \%)$ & 0 & $99(100 \%)$ \\
\cline { 2 - 5 } & V & Nil & Nil & Nil \\
\hline
\end{tabular}

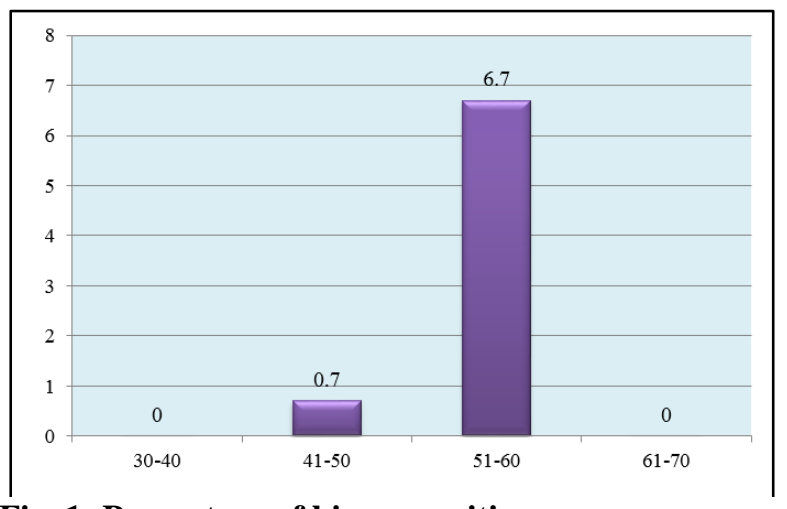

Fig. 1: Percentage of biopsy positive cases distribution with age of the patients

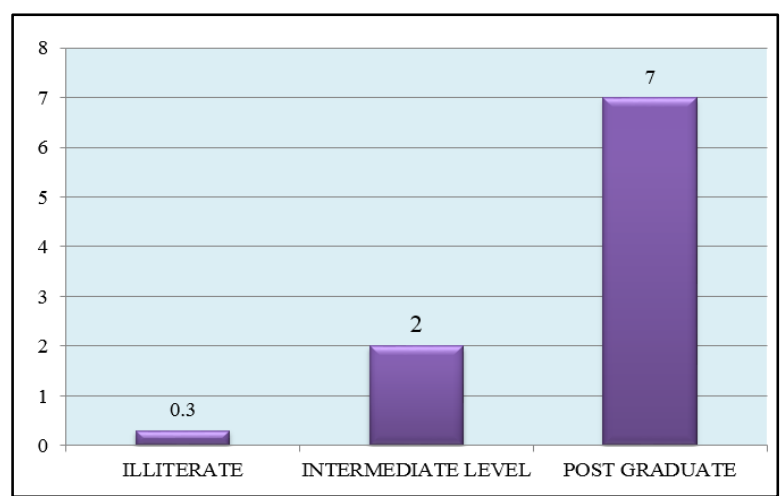

Fig. 2: Percentage of biopsy positive cases distribution with literacy status of patients

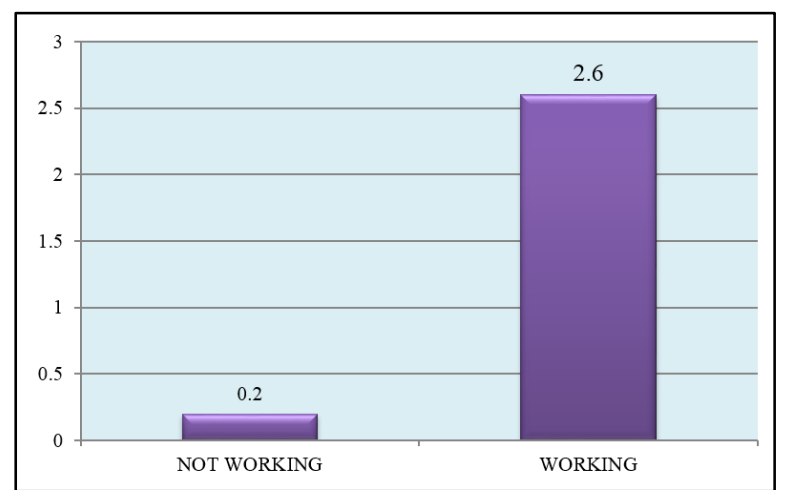

Fig. 3: Percentage of biopsy positive cases distribution with working status of patients

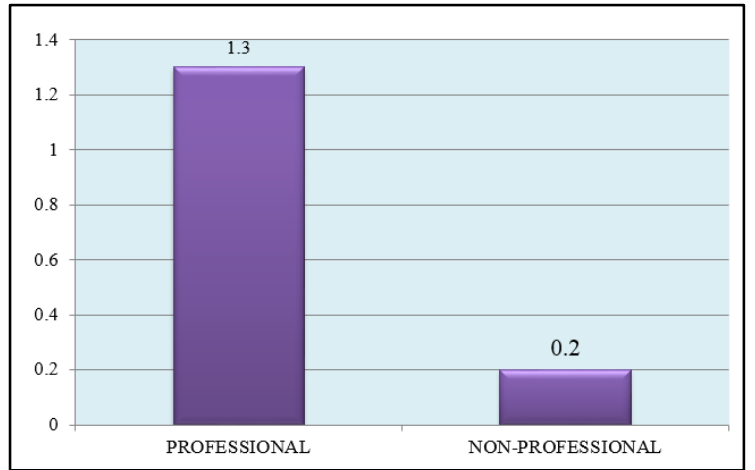

Fig. 4: Percentage of biopsy positive cases distribution with working status of the husband of patients

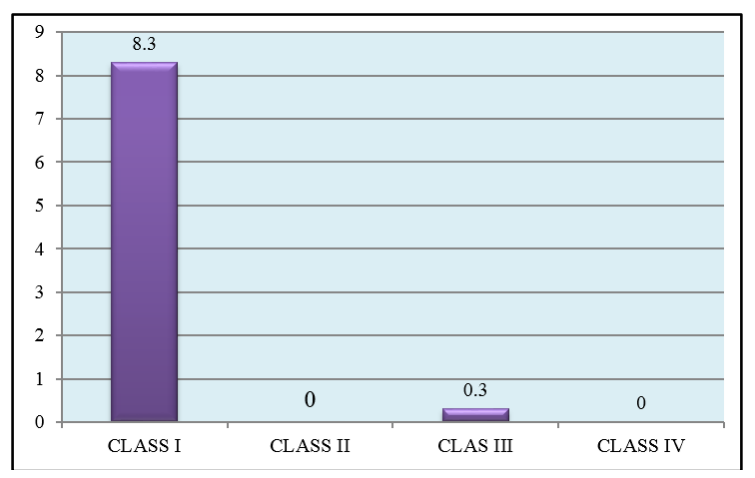

Fig. 5: Percentage of biopsy positive cases distribution with socio-economic class status of patients

\section{Discussion}

The present study showed that three biopsy positive cases were found in the age group of 41 to 50 years and one case in age group of 51-60 years. Vedantham et al. observed in their study that VIA positivity was significantly correlated with age over 60 years with an adjusted OR of 2.08 (C.I 1.25-3.47) times higher chances of developing cervical cancer compared to women in age group of 25-29 years. ${ }^{9}$

Globally it was observed that in unscreened populations, the peak risk of invasive cervical cancer occurs earlier then for most adult cancers, peaking or reaching a plateau from about $35-55$ years. ${ }^{10}$

In India the peak age for cervical cancer incidence is 55-59 years. ${ }^{11}$ A recent study done by Saurabh Bobdey, Jignasa Sathwara, Ganesh Balasubramaniam 
(2016) revealed that in India more than $85 \%$ of patients were from age group 40 years and above. ${ }^{12}$ The maximum numbers of cases were reported in 50-59 years of age group amounting to $27.37 \%$ of all cervical carcinoma cases as revealed in a three year report population based cancer registries. ${ }^{13}$

This study showed that there was significantly higher distribution of cervical biopsy positive cases amongst women with higher literacy status, i.e. at least intermediate level or higher. ${ }^{13}$ However, Vedantham et al. (2010) observed in their study that VIA positivity was significantly correlated with poor literacy status. ${ }^{9}$ Similarly Franceschi S. et al. (2003) in their multicenter case-control study in Chennai, Southern India revealed that ORs and corresponding 95\% CIs Illiteracy compared to higher education increases risk to nearly 5 times for development of cervical cancer $(\mathrm{OR} v s$. high education $=4.8){ }^{14}$

We can possibly explain our findings by there being a probability of women with higher education more likely to visit health centres for complaints related to reproductive health and thereby more likely to be screened out for CIN (cervical intra-epithelial) changes. Women with higher education are likely to have better awareness on importance of reproductive health compared to women with poor literacy status.

Table 1 showed that all the four biopsy positive cases were found amongst participants who were Hindu by religion. However the difference in the religion distribution with biopsy positive cases was not found to be statistically significant. Seema P. et al. (2003) in their meta-analysis study on social inequalities as risk factors for cervical cancer revealed that specific religious practices also modify the risk of developing cervical cancer in women following HPV infection. ${ }^{15}$ Dikshit R. et. al. (2012) in their Million Death study done in several parts of India between the years 2001 and 2003 revealed that Cervical cancer was far less common in Muslim than in Hindu women [study deaths 24 , age-standardised mortality ratio $0.68(0.64-0.71)$ vs $340,1.06(1.05-1.08)]$. Circumcision amongst Muslim men, which reduces the sexual transmission of human papilloma a virus is a likely explanation. ${ }^{16}$ Study showed that significantly higher proportion of females with positive cervical biopsy cases was amongst the women who were currently working compared to women who were not working. Arbyn M. et al. in their study in 2008 revealed that Age-specific analyses clearly indicated that cervical cancer primarily affects young adult women who are actively involved in their careers or caring for their families. ${ }^{2}$ The possible explanation of significant occurrence of cervical cancer cases amongst working women could be that these working women are more empowered to make decision related to their health and get themselves screened for cervical cancer as opposed to house-wives who do not have such decision making power related to their health needs and often face various social restriction to undergo such a screening. ${ }^{17}$

Similarly it was seen in this study that significantly higher number of CIN changes cases were seen amongst women whose husband were doing some higher professional job compared to those whose husband were working as either skilled/semiskilled/unskilled labors or unemployed. Also we found that significantly higher proportion of CIN changes were seen amongst women who belonged to better or higher socio-economic class compared to lower socioeconomic class.

Study findings are contrary to innumerable studies which show an excess of cervical cancer among low socioeconomic class women; to an extent that this considered as one of the earliest and most consistent findings of epidemiologic studies of this tumour. ${ }^{18}$ Parikh S. et al. (2003) reported in their meta-analysis of case control studies done on cervical that based on 57 studies, they found an increased risk of approximately $100 \%$ between high and low social class categories for the development of invasive cervical cancer, and an increased risk of approximately $60 \%$ for dysplasia, including carcinoma in situ. ${ }^{19}$

Thus all these studies reported a consistent increasing trend in cervical cancer incidence with various indicators of decreasing social class, including level of education, income and occupation. The main explanations advanced for the excess risk among lower socio-economic groups are related to sexual behaviour, corresponding to a greater chance to acquire and/or become chronic carriers of human papillomavirus (HPV), which is a necessary cause of cervical cancer. Less access to early diagnosis or cytological screening for cervical cancer is also likely to result in a higher incidence and mortality. Overall, an increased relative risk of dysplasia and cervical cancer with decreasing social class was observed when all studies were pooled women in the middle social class group were at approximately a $26 \%$ increased risk of cervical disease (95\% CI $17-36 \%$ ), whereas women in the lower social class were at approximately an $80 \%$ increased risk when compared to women in the upper social class (95\% CI 69-92\%). When stratified by geographical region it was found that the risk was greater in studies originating from North America and in studies originating from South America, Asia and Africa, where approximately a $100 \%$ increased risk was observed in these regions among the low social class group when compared to the high social class group. ${ }^{19}$

The possible explanation for discrepancy in our findings could be that these women who had better literacy status, who were financially independent and had better socio-economic status were more empowered to participate in the screening of cervical cancer and thus had a better chance to be diagnosed early and treated appropriately. 


\section{Conclusion and Recommendations}

Study revealed that many socio demographic variables such as increasing age, literacy status, religion, working status of participant and her husband, socio-economic status are important factors that significantly showed higher proportion of dysplasia that can influence development of cervical cancer. Also these factors are supported by several epidemiological studies as important risk factors for development of cervical cancer, specially done in low resource settings.

Lastly usage of VIA for cervical screening was an easy and convenient method to screen cases at a peripheral level and the benefit was that it required no costly lab based procedures, was quick in giving results and the patients could be immediately sent for management. Cervical screening with VIA has a good potential in helping screening population catered by low resource settings where there is scarcity of manpower, material and money.

The advent of HPV vaccines had added a new weapon in the fight against cancer of the cervix. HPV 16 and HPV -18 are the most common types a vaccine targeting these types could eliminate $75 \%$ of the cervical cancer in the country. HPV vaccines namely Gardasil and Cervarix prevent infection with HPV type 16 and 18 which are responsible for $90 \%$ of the cervical cancer cases. Gardasil also prevents HPV type 6 and 11 which causes $90 \%$ of the genital warts. Timely implementation of an affordable and effective screening strategy in developing country is thus crucial, while waiting for further improvements in HPV testing, vaccine technology, costs, and its widespread use. ${ }^{12}$ Thereby a comprehensive disease control initiative -a combination of improved screening and treatment of pre-cancerous lesions (secondary prevention) with effective HPV vaccination (primary prevention)-has the best potential to significantly reduce the burden of cancer cervix relatively soon.

Mass health education and behaviour change interventions should be conducted at the community level in order to make all women of child bearing age aware about cervical cancer and its screening. The benefits of early diagnosis and treatment must be made aware to women so that they are encouraged to take cervical cancer screening.

VIA has time proved and it is worth in cervical screening especially in low-resource settings. Staff (medical and paramedical workers and other health care providers) should be trained for VIA procedure so that they can easily conduct this test and screen maximum population and refer them to higher center for appropriate management

\section{References}

1. Dennny L. Cancer Cancer: Prevention and Treatment. Discov Med 2012;14:125-31.

2. Arbyn M., Castellsaque X., Desanjose S., et al. Worldwide burden of cervical cancer. Ann Oncol 2011;2675-86.
3. Yesle BB, Kumar AV, Kerkureet A., Sunny L.

Population based survival from cancers of breast, cervix and ovary in women in Mumbai. Asian Pac J Cancer Prevention 2004;5:308-15.

4. World Health Organization. The Global Burden of Disease: 2004 Update. Geneva: World Health Organization: 2008.

5. Hakama M, Miller AB, Day NE. Screening for Cancer of the Uterine Cervix.from the IARC Working Group on Cervical Cancer Screening and the UICC Project Group on the Evaluation of Screening Programmes for Cancer. WHO (Geneva), IARC (Lyon), and UICC (Geneva) IARC Sci Publ 1986;76:1-315.

6. Coffey P, Arrossi S, Bradley J, Dzuba I, White S, ACCP Community Involvement Affinity Group. Improving screening coverage rates of cervical cancer prevention programs: a focus on communities. Seattle: Alliance for Cervical Cancer Prevention; 2004. (issues in Depth No. 4) Available from http://www.path.org/files/RH_accp_improve_screening.p df

7. Denny L, Kuhn L, Pollacl A, Wright TCJR. Direct visual inspection for cervical cancer screening: an analysis of factors influencing test performance. Cancer 2002;94:1699-707.

8. Disease Specific Documents for XII plan. Human Papilloma Virus ICMR: High power Committee to Evaluate Performance of ICMR, 2012-2013. New Delhi, India:ICM;2014

9. Vedantham H, Silver MI, Kalpana B et al. Determinants of VIA (Visual inspection of the cervix After Acetic Acid Application) positivity in cervical cancer screening of women in a peri-urban area in Andhra Pradesh, India. Cancer Epidemiological Biomarkers and Prevention 2010;19:13.

10. WHO. Cervical cancer screening in Developing Countries: Report of a WHO consultation. World Health Organization, 2002. Available from http://www.who.int/cancer/media/en/cancer_cervical_373 21.pdf

11. World - both sexes estimated incidence by age. Available from:

http://www.globocan.iarc.fr/old/age_specific_table_r.asp?

12. Saurabh Bobdey, Jignasa Sathwara, Aanchal Jain, Ganesh Balasubramaniam. Burden of cervical cancer and role of screening in India. Ind J Medi Pediat Oncol 2016;37(4):278-85.

13. Three-Year Report of Population Based Cancer Registries 2012-2014. Bengaluru: National Centre for Disease Informatics and Research National Cancer Registry Program (ICMR); 2016. Mar, Incidence, Distribution, Trends in Incidence Rates and Projections of Burden of Cancer; pp. 1-15.

14. Franceschi S, Rajkumar T, Vaccarella, et al. Human papillomavirus and risk factors for cervical cancer in Chennai, India: a case-control study. Int J Cancer 2003;107:127-33.

15. Seema P, Paul B, Boffetta P. Meta-analysis of social inequality and the risk of cervical cancer. Int J Can 2003;105:687-91.

16. Dikshit R, Gupta PC, Ramasundarahettige C. et al. Cancer mortality in India: a nationally representative survey. The Lancet 2012:1807-16.

17. Ronco G, Segnan N, Ponti A. Who has Pap tests? Variables associated with the use of Pap tests in absence of screening programmes. Int J Epidemiol 1991;20:34953. 
18. Schiffman MH, Brinton LA, Devesa SS, Fraumeni JF Jr. Cervical cancer. In: SchottenfeldD, Fraumeni JF Jr, eds. Cancer epidemiology and prevention, 2nd ed. Oxford: Oxford University Press, 1996;2:1090-116

19. Parikh S, Brennan P, Boffetta P. Meta-analysis of social inequality and the risk of cervical cancer. Int $J$

Cancer 2003;105: 687-91. 